\title{
JURISPRUDENCIA AMBIENTAL EN LA RIOJA (PRIMER SEMESTRE 2019)
}

\author{
LuCía MuÑOZ BENITO \\ Profesora interina de Derecho administrativo \\ Universidad de La Rioja
}


SUMARIO: 1. Introducción. 2. Ámbito de aplicación de la licencia ambiental: ¿modificación sustancial? 3. El impuesto sobre el valor de la producción de la energía eléctrica: cuestión de inconstitucionalidad de la Ley 15/2012, de 27 de diciembre, de medidas fiscales para la sostenibilidad energética. 4. Sanciones en materia medio ambiental. 4.1. Incumplimiento de las condiciones establecidas en la autorización ambiental integrada 4.2. Agricultura ecológica. 4.3. Caza

\section{INTRODUCCIÓN}

En el periodo objeto de recopilación el Tribunal Superior de Justicia de La Rioja ha dictado ocho sentencias en materia medio ambiental de las que se va a dar cuenta en esta crónica.

De estas sentencias, seis están relacionadas con sanciones impuestas en diferentes ámbitos medio ambientales. Las dos restantes tienen que ver con el ámbito de aplicación de la licencia ambiental y el impuesto sobre el valor de la producción de la energía eléctrica.

\section{2. ÁmBito DE APLICACIÓN DE LA LICENCIA AMBIENTAL: ¿MODIFICACIÓN SUSTANCIAL?}

La sentencia del TSJ de la Rioja núm. 69/2019, de 20 de febrero resuelve un recurso de apelación promovido por el Ayuntamiento de Manjarrés y la Consejería de Agricultura, Ganadería y Medio Ambiente del Gobierno de la Rioja contra la sentencia núm. 198/2018, de 3 de julio de 2018 del Juzgado de lo Contencioso-Administrativo núm. 1 de Logroño.

La sentencia del Juzgado de lo Contencioso-Administrativo había estimado el recurso presentado por un particular contra la desestimación presunta por silencio de la petición de revisión de oficio dirigida al Ayuntamiento para que declarara nula la licencia de obras y sus prórrogas concedida a la Granja "EI Carrascal" S.L., para impermeabilizar unas balsas de purines porque el Ayuntamiento debió exigir la obtención de licencia ambiental. El juzgador de instancia se limitó, dada la naturaleza revisora de la jurisdicción, a ordenar al Ayuntamiento a que iniciara el procedimiento de revisión previsto en el entonces vigente art. 102 de la Ley 30/1992 y dictase la oportuna resolución expresa diciendo si había o no nulidad. 
El Tribunal Superior de Justicia va más allá y entra de lleno en el análisis de si el proyecto de impermeabilización de la balsa de purines necesita o no licencia ambiental. Con base en la ya derogada Ley 5/2002, de 8 de octubre, de Protección del medio Ambiente de La Rioja, en especial sus arts. 6.c) y 25, el TSJ entiende que lo único que hay que dilucidar es si la impermeabilización de la balsa de purines supone una modificación sustancial de la instalación, lo cual llevaría a la conclusión de que es necesario someterla a licencia ambiental.

De este modo, teniendo en cuenta el art. 53 del Reglamento de desarrollo de la Ley, aprobado por Decreto 62/2006, de 10 de noviembre, que es el que recoge qué se entiende por modificación sustancial, el Tribunal entiende que la modificación para la que se solicita la licencia de obras no encaja en ninguno de los supuestos previstos en los apartados a) y b) del citado artículo ${ }^{1}$, por lo que, en virtud del apartado c), tendría que haber sido el órgano ambiental municipal quien decidiera si la modificación era sustancial o no "atendiendo a las características de la instalación o actividad y su previsible incidencia sobre las personas, bienes y el medio ambiente".

Dado que el Ayuntamiento, al conceder la licencia de obras, no consideró que la modificación se encontraba entre las previstas en el art. 53, en el estudio de la misma se cumplieron todos los requisitos legales, aunque cabe resaltar que el propio Tribunal destaca la celeridad que supone conceder una licencia en un día, como fue el caso.

Con base en lo anterior, el TSJ entiende que no se puede considerar que el acto sea nulo de pleno derecho y estima el recurso, dando la razón al Ayuntamiento.

\footnotetext{
${ }^{1}$ Estos supuestos son: a) que la modificación suponga un incremento del $20 \%$ del volumen o de la superficie de la instalación o del $15 \%$ del volumen de la producción y b) que tengan una incidencia significativa en la calidad o capacidad regenerativa de los recursos naturaleza del área territorial donde se ubique.
} 


\section{EL IMPUESTO SOBRE EL VALOR DE LA PRODUCCIÓN DE LA} ENERGÍA ELÉCTRICA: CUESTIÓN DE INCONSTITUCIONALIDAD DE LA LEY 15/2012, DE 27 DE DICIEMBRE, DE MEDIDAS FISCALES PARA LA SOSTENIBILIDAD ENERGÉTICA

En el recurso que resuelve la Sentencia del TSJ de La Rioja núm. 72/2019, de 21 de febrero, la demandante había instado el planteamiento de una cuestión de inconstitucionalidad en relación con diversos preceptos de la Ley 15/2012, de 27 de diciembre, de medidas fiscales para la sostenibilidad energética, que regulan el impuesto estatal sobre el valor de la producción de la energía eléctrica. Pero esta pretensión se rechaza con apoyo en el ATC 69/2018, de 20 de junio, que ya había inadmitido una cuestión similar planteada por la Sala de lo Contencioso-Administrativo del Tribunal Supremo. En síntesis, de este pronunciamiento se desprende que "la doble imposición entre el impuesto sobre el valor de la producción de la energía eléctrica y el IAE no vulnera per se ningún precepto constitucional. Para alcanzar esta conclusión no es preciso abordar en qué grado aquel presenta una finalidad extrafiscal y si esta predomina o no sobre su función recaudatoria, lo que sólo cobraría sentido desde la premisa de una prohibición de doble imposición que, según se ha indicado, solo existe como regla de coordinación entre los tributos propios de las Comunidades Autónomas y los estatales y locales y no de estos últimos entre sí".

\section{SANCIONES EN MATERIA MEDIO AMBIENTAL}

Como ya apuntábamos en la introducción, en el periodo recopilado se han dictado seis sentencias en diferentes procesos sancionadores relativos a la actividad de policía ambiental, la agricultura ecológica y la caza.

\subsection{Incumplimiento de las condiciones establecidas en la autorización ambiental integrada}

La Sentencia del TSJ de La Rioja núm. 261/2018, de 13 de septiembre resuelve un recurso contencioso-administrativo presentado contra la resolución del Consejero de Agricultura, Ganadería y Medio Ambiente de la Comunidad 
Autónoma de La Rioja de 14 de noviembre de 2017 que desestimaba el recurso de alzada interpuesto contra la resolución del Director General de Calidad Ambiental y Agua de 3 de octubre de 2017 imponiendo una sanción de 35.000 euros de multa.

Los hechos del caso giran en torno a varios incumplimientos tanto del Real Decreto 1481/2001, de 27 de diciembre, por el que se regula la eliminación de residuos mediante depósito en vertedero como, especialmente, del contenido de la autorización ambiental integrada del vertedero de residuos no peligrosos de Arnedo concedida por Resolución núm. 358, de 2 de diciembre de 2013 al promotor del vertedero y recurrente en el caso que nos ocupa. El promotor, que ya contaba con una autorización ambiental integrada de fecha 27 de noviembre de 2007, había solicitado una nueva autorización para la adecuación de un nuevo vaso de vertido.

Los incumplimientos que fueron detectados tras las inspecciones realizadas al vertedero y recogidos en el texto de la sentencia son los siguientes:

- En primer lugar, el vertedero contaba, en la fecha de la concesión de la nueva autorización integrada con tres vasos de vertido: el vaso de vertido 0 , fuera de operación y usado de manera temporal hasta que el vaso de vertido 1 quedó acondicionado; el vaso de vertido 1, que no estaba en operación y se encontraba en fase de clausura; y el vaso de vertido 2, que se encontraba en fase de explotación.

En este caso los hechos denunciados fueron que la clausura de los vasos 0 y 1 se estaba llevando a cabo sin la preceptiva solicitud a la Administración y, en consecuencia, sin seguir el procedimiento legal. Además, en la fecha de inspección llevada a cabo el 11 de noviembre de 2016 y posteriormente el 12 de julio de 2017 se detectó que tampoco el vaso 2 se encontraba sellado, a pesar de que había un proyecto de sellado y se habían iniciado las obras.

En este sentido, según el RD 1481/2001 y la autorización ambiental integrada, debería existir, en la fecha de los hechos, una resolución del Director General en la que constaran que las obras de clausura de los 
vasos 0,1 y 2 habían finalizado, se había comprobado la clausura realizada y establecido las condiciones de post-clausura, todo lo cual no existía.

- En segundo lugar, se detectó el incumplimiento del proyecto conforme al cual se concedió la autorización ambiental integrada por contar con insuficientes medios personales en relación con los que se señalaban en el proyecto. Asimismo, el personal tampoco contaba con una formación suficiente respecto al plan de explotación del vertedero.

- En tercer lugar, no existía control a la entrada de la instalación.

- Por último, la explotación del vaso 3 del vertedero, a la que se refería la Resolución de autorización ambiental integrada de 2 de diciembre de 2013, no se estaba realizando conforme al proyecto por cuanto se infringían las medidas referidas a la colocación de residuos, estabilidad de la masa y estructuras asociadas.

Estos hechos fueron considerados por el órgano sancionador como constitutivos de una infracción tipificada como grave en el art. 53.2.2.a) de la ya derogada Ley 5/2002, de 8 de octubre, de Protección del Medio Ambiente de la Rioja y, en consecuencia, se impuso la sanción de multa de 35.000 euros.

El recurrente alegó como motivos de impugnación los siguientes: en primer lugar, la nulidad del expediente por entender que no había tenido derecho a su acceso y a que sus alegaciones fueran respondidas conforme al art. 53 de la Ley 39/2015; en segundo lugar, la vulneración del principio de presunción de inocencia por entender que la actividad inspectora se apoyaba en meras conjeturas y opiniones subjetivas, siendo necesario que del contenido de las actas inspectoras se adquiera la convicción respecto a la conducta reprochada y a la culpabilidad del acusado; por último, la vulneración del principio de proporcionalidad por entender que ni la trascendencia de los hechos denunciados, ni el grado de imputabilidad de los mismos, ni las circunstancias concurrentes justificaban la sanción en un importe superior al mínimo. 
Todos ellos son desestimados por el TSJ al entender que había quedado acreditado el acceso al expediente y la realización de alegaciones; éstas, junto con el análisis del expediente, no habían desvirtuado los hechos por los que había sido sancionado; y, por último, la sanción se graduó teniendo en cuenta el beneficio obtenido y los incumplimientos y, en consecuencia, entiende que no es desproporcionada.

\subsection{Agricultura ecológica}

En el proceso que resuelve la Sentencia del TSJ núm. 57/2019, de 14 de febrero se discute la conformidad a Derecho de varias sanciones impuestas a una sociedad productora de miel ecológica en aplicación de la Ley 5/2005, de 1 de junio, de los sistemas de protección de la calidad agroalimentaria en la Comunidad Autónoma de La Rioja. El debate procesal gira fundamentalmente en torno a cuestiones de hecho cuya apreciación por la Sala determina la estimación parcial del recurso. Así, confirma las multas por la comisión de una infracción muy grave (consistente en la expedición de mercancías que no correspondan a las características de calidad mencionadas en sus medios de comercialización) y por otra infracción grave (consistente en la venta de la miel controvertida durante la vigencia de las medidas cautelares adoptadas). Pero anula, en cambio, una tercera multa por no estar acreditada la obstrucción a las labores de inspección y también la sanción accesoria de baja definitiva en el Registro de elaboradores de productos ecológicos. En relación con esto último, el Tribunal razona que "las consecuencias que conlleva la baja definitiva en el Registro (exclusión de los infractores, y como consecuencia la pérdida de sus derechos inherentes a la denominación o marca) exige un esfuerzo de motivación por parte de la Administración, determinando su ausencia que se desconozcan las razones por las que se opta por acordar la sanción accesoria y, por tanto, si ésta es proporcionada". 


\subsection{Caza}

Son cuatro las sentencias dictadas por el Tribunal Superior de Justicia sobre sanciones en el ámbito de la caza por aplicación de la Ley 9/1998, de 2 de julio, de Caza de La Rioja.

La Sentencia núm. 268/2018, de 20 de septiembre, desestima el recurso contencioso-administrativo interpuesto contra la resolución del Consejero de Agricultura, Ganadería y Medio Ambiente de fecha 3 de octubre de 2017, por la que se imponía una sanción de multa de 500 euros y, como sanciones accesorias, la pérdida de licencia de caza en vigor e inhabilitación para obtenerla por un periodo de 6 meses. La resolución entendía que los hechos denunciados -transportar un arma cargada en zona de seguridad de un camino público, efectuando un disparo y causando la muerte de un perro- daban lugar a una infracción grave tipificada en el art. 82.1 de la citada Ley².

El demandante alegaba en su escrito de demanda la vulneración del principio de presunción de inocencia por entender que la denuncia por parte de la Guardia Civil carecía de valor y no quedaba probado que el denunciado deambulara con el arma cargada por una zona de seguridad.

El TSJ desmonta este motivo de impugnación con base en lo siguiente: en primer lugar, en virtud del art. 21 de la Ley, se consideran zonas de seguridad, entre otros, los caminos rurales; en segundo lugar, el art. 95 de la Ley recoge la presunción de veracidad de las denuncias de los agentes de la autoridad; y, por último, de las propias declaraciones del demandante se infiere que la escopeta estaba cargada y la fijación del punto donde se produjo la muerte del animal demuestran que los hechos tuvieron lugar en un camino y, por tanto, zona de seguridad a efectos de la Ley. Todo ello, dice el Tribunal, "es suficiente para desvirtuar la presunción de inocencia" y, en consecuencia, para desestimar el recurso.

La Sentencia núm. 270/2018, de 27 de septiembre, desestima el recurso contencioso-administrativo interpuesto contra la Resolución de 5 de julio de 2017 del Consejero de Agricultura, Ganadería y Medio Ambiente que

\footnotetext{
2 "Tendrán la consideración de infracciones graves las siguientes: 1. Cazar o transportar armas u otros medios de caza listos para su uso en zonas de seguridad, sin autorización, aun cuando no se hubiera cobrado pieza alguna".
} 
desestimaba el recurso de alzada interpuesto contra la Resolución de 19 de abril de 2017, que imponía una multa de 903 euros, la pérdida de la licencia de caza y la inhabilitación para obtenerla por periodo de un año y medio, así como el pago de manera solidaria, de los daños y perjuicios ocasionados al titular del acotado.

En este caso los hechos tenían que ver con la práctica de la caza en un coto sin autorización para ello, el ejercicio sin autorización de la modalidad de caza denominada de gancho y la ocultación a los agentes de la autoridad de la pieza abatida. Estos hechos, según las resoluciones administrativas que se impugnaban, fueron constitutivos de dos infracciones de carácter grave, a saber: la prevista en el art. 82.19 (cazar sin autorización) y la prevista en el apartado 29 del mismo artículo de la Ley (negarse a entregar las piezas de caza obtenidas durante la comisión de una infracción tipificada en la Ley a los agentes de la autoridad).

Al igual que en la Sentencia reseñada anteriormente, la parte demandante alega la vulneración del principio de inocencia por entender que no hay una prueba incriminatoria suficiente frente a él.

EI TSJ resuelve de nuevo con base en el art. 95 de la Ley que, al igual que el art. 75.2, recoge la presunción de veracidad de las denuncias formuladas por los agentes de la autoridad, salvo prueba en contrario. Así, los hechos descritos por los agentes en la denuncia sirven como prueba suficiente para acreditar que lo que se estaba practicando era la modalidad denominada "ganchos de caza mayor", prevista en el art. 66 del Decreto 17/2004, de 27 de febrero, por el que se aprueba el Reglamento de Caza de La Rioja y que, como recoge el texto de la Sentencia, "consiste en una cacería colectiva practicada con ayuda de perros y/o batidores con el fin de levantar piezas de caza mayor existentes en una determinada extensión de terreno y obligarlas a dirigirse hacia los cazadores que en número máximo de nueve y provistos de medios autorizados, fueron previamente colocados en puestos fijos".

Por tanto, en virtud de la presunción de veracidad de la denuncia efectuada por los agentes de la autoridad y de la prueba testifical, que desacredita las alegaciones del recurrente, queda desvirtuada la presunción de inocencia y se acredita su participación en una modalidad de caza no autorizada. 
También de fecha 27 de septiembre es la Sentencia núm. 272/2018, desestimatoria del recurso contencioso-administrativo interpuesto contra la Resolución de 25 de septiembre de 2017 del Consejero de Agricultura, Ganadería y Medio ambiente por la que desestima el recurso de alzada interpuesto contra la Resolución de 10 de julio de 2017, que imponía una multa de 1500 euros, la pérdida de la licencia de caza y la inhabilitación para obtenerla por un periodo de un año, así como la prohibición de obtener permisos de caza en terrenos cinegéticos gestionados por la Comunidad Autónoma de La Rioja durante un plazo de dos años.

Las Resoluciones impugnadas consideraban probado que el recurrente había cazado incumpliendo las condiciones de la autorización de caza en batida en la Reserva Regional por hacerlo en un Polígono distinto para el que había sido autorizado y que estos hechos eran constitutivos de la infracción grave recogida en el art. 82.19 de la Ley de Caza (cazar sin autorización).

El demandante fundamentaba su recurso en tres motivos: en primer lugar, la nulidad o anulabilidad del procedimiento sancionador por no resolverse sobre todas las cuestiones alegadas; en segundo lugar, la nulidad de actuaciones o la anulabilidad por cuanto la propuesta de resolución no resuelve el hecho fundamental de que el Guarda de la Reserva permitió realizar la batida; y, en tercer lugar, la falta de aplicación de los principios del proceso penal extensibles al procedimiento administrativo sancionador.

En este sentido, recuerda el TSJ que solo cabe la nulidad del procedimiento administrativo si se ha producido indefensión al interesado, lo cual se desconoce porque el demandante no dice qué cuestiones del procedimiento no han sido resueltas. Por otro lado, si bien en la propuesta de resolución no se trata la cuestión del permiso del Guarda, esto no produce indefensión al recurrente porque pudo combatir la razón por la que se consideraba cometida la infracción y, además, el recurso de alzada insiste en que la batida no fue autorizada por nadie, demostrando que el recurrente mintió. Por último, considera el Tribunal que sí se han aplicado plenamente los principios que rigen el proceso penal al procedimiento administrativo sancionador, no habiéndose incurrido en vulneración de ninguno de ellos. Por todo ello el TSJ desestima el recurso contencioso-administrativo. 
Por último, la Sentencia del TSJ de La Rioja núm. 70/2019, de 21 de febrero, al igual que las anteriormente citadas, confirma la sanción de multa de 3501 euros y pérdida de la licencia de caza e inhabilitación para obtenerla por un periodo de 3 años y 6 meses impuesta por resolución de 6 de abril de 2018 por el Consejero de Agricultura, Ganadería y Medio Ambiente del Gobierno de La Rioja.

En este caso los hechos fueron que el denunciado se encontraba en el monte de madrugada junto con su vehículo, en el cual había un rifle fuera de la funda y munición preparada en el asiento del copiloto, así como una escopeta enfundada junto con 220 cartuchos y varios cuchillos y machetes en el maletero. Estos hechos fueron considerados como constitutivos de dos infracciones muy graves tipificadas en los arts. 81.1. y 82.14 de la Ley33.

El Tribunal, de nuevo considerando insuficientes las alegaciones del recurrente, que manifestaban que se encontraba buscando un teléfono móvil, apela a la presunción de veracidad de las denuncias formuladas por los agentes de la autoridad prevista en el art. 95 de la Ley para desestimar el recurso.

\footnotetext{
${ }^{3}$ Estas infracciones consisten, respectivamente, en "cazar o transportar armas $u$ otros medios de caza listos para su uso de noche auxiliándose con los focos de su vehículo a motor o con cualquier otro dispositivo que emita luz artificial o facilita la visión nocturna" y "cazar o transportar armas u otros medios de caza listos para su uso, en terrenos cinegéticos, sin el correspondiente permiso del titular, aun cuando no se haya cobrado pieza alguna".
} 